\title{
Briefe württembergischer Fürsten aus dem 16. Jahrhundert aus einer Sammlung in St.Petersburg
}

\author{
Von Tamara N. Tatcenko
}

Im Wissenschaftlich-Historischen Archiv am St. Petersburger Institut für Geschichte / Russische Akademie der Wissenschaften (WHA St. Pt. Institut f. Geschichte) werden 15 nicht veröffentlichte und bislang unbekannte Briefe von Mitgliedern des württembergischen Fürstenhauses aus dem 16. Jahrhundert aufbewahrt ${ }^{1}$. Sie gehören hier zum Archivbestand 25 „Deutschland. Akten und Briefe der Feudalherren vom 14. bis 19. Jahrhundert“, der einen Teil der großen Handschriftensammlung des bekannten russischen Historikers und Sammlers Prof. Nikolaj Petrovič Lichačev (1862-1936) darstellt. Von 1902 bis 1914 war Lichačev als Stellvertretender Direktor der Kaiserlichen Öffentlichen Bibliothek in St.Petersburg tätig und hielt auch Vorlesungen über Historische Hilfswissenschaften an der St. Petersburger Universität. Sein Ziel war es, eine große Sammlung mit Denkmälern der Schriftlichkeit aus verschiedenen Kulturen und Epochen zu bilden und dadurch eine Quellenbasis für vergleichende Studien über Diplomatik, Paläographie, Sphragistik usw. zu schaffen. Aus diesem Grund pflegte Lichačev enge und ständige Kontakte zu wichtigen Antiquariaten in Westeuropa, wo er mit eigenen Mitteln zwischen 1892 und 1914 zahlreiche Handschriften aus dem Mittelalter und der Frühen Neuzeit erwerben konnte. Zum langjährigen Kunden wurde er auch bei deutschen Antiquariatshäusern wie „Leo Liepmannssohn“ (Berlin), „List und Franke“ (Leipzig), „Karl Ernst Henrici“ (Berlin) u.a., wo er deutsche Akten und Briefe für seine Sammlung kaufen konnte. Bei einigen von ihnen sind Belege dafür erhalten, darunter für sieben von 15 Briefen des 16. Jahrhunderts aus Württemberg. Die Umschläge mit diesen Schriftstücken tragen entsprechende Vermerke der Antiquare und enthalten Ausschnitte aus den Auktionskatalogen.

${ }^{1}$ Ein Großteil der Briefe wurde bereits in einer russischen Publikation vorgestellt. Vgl. Tamara N. Tatcenko, Pisma XVI w. herzogow Würtembergskich w kollekzii N.P. Lichačeva. Zapadnojewropejskaja sekzija archiwa Sankt-Peterburgskogo Instituta istorii RAN, in: Wspomogatelnyje istoricheskije diszipliny 30 (2007) S.311-332. Herrn Prof. Dr. Peter Rückert schuldet die Autorin aufrichtigen Dank für seine vielseitige Hilfe bei der Drucklegung des Beitrags. 
Die betreffenden Briefe umspannen den Zeitabschnitt von 1552 bis 1592, ihre Absender sind folgende Württembergischen Herzöge und Grafen: Herzog Christoph (1515-1568) $)^{2}$, seine Frau Anna Maria, geb. Markgräfin von Brandenburg-Ansbach (1526-1589)3 , Herzog Ludwig (1554-1593) ${ }^{4}$, Graf Georg von Württemberg-Mömpelgard (1498-1558) ${ }^{5}$, Graf Friedrich von Württemberg-Mömpelgard, später Herzog von Württemberg (1557-1608) $)^{6}$ Die Briefe sind an verschiedene Empfänger gerichtet: gleichgestellte Personen fürstlichen Standes, städtische oder fürstliche Regierungen, Amtsträger der eigenen Verwaltung sowie weitere untergeordnete Personen. Nach der gängigen Klassifikation der Aktenkunde der Neuzeit gehören sie zu der Kategorie der „geschlossenen Schreiben“ und enthalten vor allem Anweisungen, Mitteilungen, Ersuchen, Bitten ${ }^{7}$.

Die Schreiben sind Werke der fürstlichen Kanzleien, wo sie nach einem bestimmten mehr oder weniger vollständigen Formular (Intitulatio am Anfang oder am Ende des Textes / Anrede / Antritt / Narratio / Dispositio / Schlusscourtoisie / Unterschrift) von Kanzleisekretären verfasst und von professionellen Schreibern auf Papier ausgeführt worden waren. Es gibt in dieser Reihe nur eine Ausnahme: ein eigenhändiges Schreiben von Herzogin Anna Maria an ihren Bruder, das mit einer schwer lesbaren, von vielen individuellen Eigenheiten geprägten Schrift geschrieben ist. Sonst sind alle Briefe in der sogenannten deutschen gotischen Kurrentschrift der Neuzeit gehalten. Diese in deutschen Kanzleien damals übliche Schriftart zeigt die bekannten Eigenschaften der Kurrentschrift mit ihren feinen und eleganten Schlingen, Windungen, Schnörkeln und Schleifen.

Die zu unterschiedlichen Zeiten zufällig angekauften Schriftstücke bilden keinen einheitlichen Überlieferungskomplex. Sie verteilen sich ungleichmäßig über die zweite Hälfte des 16. Jahrhunderts und sind inhaltlich ganz aus ihren Kontexten herausgerissen. Sie ähneln winzigen Mosaiksteinen, die zufällig aus einem riesigen Bild der deutschen Geschichte des 16. Jahrhunderts herausfielen und sich nun wieder zu einer kleinen verstreuten Gruppe von Handschriften württembergischen Ursprungs fanden. Immerhin geben sie auch in dieser Form Zeugnis über mehr oder weniger bedeutende Ereignisse deutscher und württembergischer Geschichte ihrer Zeit, und als authentische Überlieferungsträger besitzen sie hierfür besondere Bedeutung.

So teilt Herzog Christoph in seinem Brief vom 2. Dezember 1552 an den Grafen von Oettingen das Datum des überraschenden Vertrags zwischen dem Kaiser und

2 Briefe 1, 2, 5, 6 .

3 Brief 7.

${ }^{4}$ Briefe 8, 9, 10, 12, 14.

5 Briefe 3, 4.

6 Briefe 11, 13, 15.

7 Jürgen Kloosterhuis, Amtliche Aktenkunde der Neuzeit. Ein hilfswissenschaftliches Kompendium, in: Archiv für Diplomatik 45 (1999) S. 465-563, hier S. 467-468. 
dem Markgrafen von Brandenburg-Kulmbach mit, der kurz zuvor geschlossen worden war (Nr.1). Dabei ging es um Markgraf Albrecht Alkibiades, der seine aktive Teilnahme am damaligen Fürstenaufstand gegen den Kaiser zum Anlass machte, Gebiete der benachbarten geistlichen Fürsten zu verwüsten und sich daran zu bereichern. Die Tatsache, dass Karl V. das Bündnis mit dem notorischen Landfriedensbrecher schloss, stieß überall auf Empörung und schadete wesentlich der Autorität des Kaisers ${ }^{8}$. Das Thema war also im Gespräch, und so wundert man sich nicht, dass sich der Graf von Oettingen für das genaue Datum des Vertrags interessierte, und Herzog Christoph Bescheid wusste.

Weitere Ereignisse, die in Verbindung mit Markgraf Albrecht Alkibiades stehen, kommen auch im Schreiben von Herzog Christoph vom 20. September 1554 vor (Nr.2). Christoph gibt eine Anweisung an die Stadt Bietigheim, sich an der Bezahlung der Kriegssteuer zugunsten der fränkischen Bischöfe, die unter den Überfällen von Albrecht Alkibiades im Markgräflerkrieg besonders gelitten hätten, zu beteiligen. Dabei bezieht er sich auf einen Beschluss des Reichskammergerichts und den Willen des Kaisers, der über Albrecht Alkibiades inzwischen die Acht ausgesprochen hatte.

Noch eine Spur des Markgrafen findet sich in Herzog Christophs Brief vom 21. Juli 1567 an seinen Schwager Georg Friedrich, Markgraf von BrandenburgAnsbach-Kulmbach (Nr.6). Dieser war Erbe von Albrecht Alkibiades und erbte dadurch nicht nur die Markgrafschaft Brandenburg-Kulmbach, sondern auch die Schulden des geächteten Markgrafen. Während des Markgräflerkrieges hatte Albrecht Alkibiades zur Finanzierung seiner Söldner mehrere Darlehen von Landgraf Georg von Leuchtenberg (1502-1555) genommen, die er nicht mehr zurückbezahlen konnte9. Nun stellten die Erben des Landgrafen 1567 erneut Anforderungen auf Rückzahlung dieser Schulden. Herzog Christoph wirkte in dieser Sache als Unterhändler. Ihm gelang es, den Streit zu schlichten, und beide Seiten einigten sich auf eine bestimmte Abfindungssumme ${ }^{10}$.

Um Schuldsachen geht es auch im Schreiben des Grafen Friedrich von Württemberg-Mömpelgard vom 28. April 1585 an die Stadt Bern (Nr.11). Friedrich war damals Herr über den linksrheinischen württembergischen Besitz. Er bittet die Berner um die Erlaubnis für Gräfin Isabella von Challant, die Grafschaft Valangin mit seiner Hilfe wiedereinzulösen. Durch den Erwerb der Grafschaft, die zwischen Bern und Mömpelgard lag, wollte Graf Friedrich seinen herrschaftlichen Besitz abrunden. Die Erbin Graf Reinhards von Challant, der 1537 seine Grafschaft Valangin an die Stadt Bern verpfändet hatte, war bereit, die Einlösungsrechte an

${ }^{8}$ Horst RaBe, Deutsche Geschichte 1500-1600. Das Jahrhundert der Glaubensspaltung, München 1991, S. 440.

9 Franz Michael Wittmann, Geschichte der Landgrafen von Leuchtenberg. Dritte Abteilung, München 1852, S. 18, 488.

10 Ebd. 
den Grafen von Württemberg-Mömpelgard zu verkaufen ${ }^{11}$. Es ist bekannt, dass Graf Friedrich für den Erwerb der Grafschaft mehrere Kredite nehmen wollte; nach langen Verhandlungen sollte sein Plan jedoch scheitern ${ }^{12}$.

In einigen Briefen spiegelt sich auch die zeitgenössische Konfessionalisierungspolitik wider. Als Beispiel dafür sei ein Brief von Herzog Ludwig an seine Kirchenräte vom 6. November 1581 angesprochen (Nr.10). Ludwig überschickt diesen neue Kirchenlieder, mit der Bitte, sie zu prüfen und den Autor dementsprechend bezahlen zu lassen. Bekanntlich war Herzog Ludwig ein frommer Lutheraner, der seine Landeskirche im lutherischen Sinne weiter ausbauen wollte. Nach der Unterzeichnung der Konkordienformel wurden 1582 die Große Kirchenordnung neu aufgelegt und 1583 das erste Gesangbuch für das Herzogtum herausgegeben ${ }^{13}$. Das betreffende Schreiben zeigt Herzog Ludwig als Landesherrn, der sich persönlich um seine Kirche kümmerte.

Weit dramatischer als im Herzogtum verlief die lutherische Konfessionalisierung in der württembergischen Grafschaft Mömpelgard. Zwar haben die württembergischen Landesherren das Luthertum dort gegen die Anhänger der Calvinisten durchgesetzt, doch mussten sie, umgeben von den habsburgischen und französischen Ländern, hier ihre konfessionelle Ausrichtung weiter gefährdet sehen. Diese Stimmung kommt in einem Brief Graf Friedrichs vom 1. September 1588 an einen nahe stehenden Fürsten zum Ausdruck (Nr.13). Friedrich hatte in seinem Hausarchiv einen Bericht über den Überfall der Lothringer auf die Grafschaft Mömpelgard von 1440 gefunden. Vermutlich bezog sich dieser auf die Besetzung Mömpelgards durch französische Truppen unter Dauphin Ludwig im Jahre 1444 ${ }^{14}$. Die Worte fast ein gleichmessiger feindtlichen einfall lassen keinen Zweifel, dass sich Graf Friedrich dabei an den gerade Ende 1587 / Anfang 1588 erfolgten Überfall der französischen Armee unter der Führung des Henri de Guise auf Mömpelgard erinnerte. Dieser galt als Rache für Friedrichs Unterstützung für die Hugenotten ${ }^{15}$. Dass Friedrich für die Sache der Evangelischen weiter warb und im Kontakt mit

11 Johann Jakob Hоттіnger, Die Schweiz in ihren Ritterburgen und Bergschlössern, Bd.2, Chur 1830, S. 46, 56.

12 HStA Stuttgart A 55 Bü 3, A 55 Bü 5. Zu Herzog Friedrich von Württemberg siehe des Weiteren die einschlägigen Beiträge in: Hofkultur um 1600. Die Hofmusik Herzog Friedrichs I. von Württemberg und ihr kulturelles Umfeld, hg. von Joachim KREMER/Sönke LOREnz/Peter RüCKert (Tübinger Bausteine zur Landesgeschichte, Bd.15), Ostfildern 2010.

${ }^{13}$ Die Evangelischen Kirchenordnungen des XVI. Jahrhunderts, Bd.16: Baden-Württemberg II. Herzogtum Württemberg, bearb. von Sabine Arend, Tübingen 2004, S. 71.

${ }_{14}$ Rainer BABEL, Mömpelgard zwischen Frankreich und dem Reich vom 16. bis zum 18. Jahrhundert, in: Württemberg und Mömpelgard. 600 Jahre Begegnung, hg. von Sönke LORENZ/Peter RüCKeRT (Schriften zur südwestdeutschen Landeskunde, Bd. 26), LeinfeldenEchterdingen 1999, S. $285 \mathrm{f}$.

15 Juliane Krieninger-Babel, Friedrich I. von Württemberg als Regent der Grafschaft Mömpelgard (1581-1593), in: Württemberg und Mömpelgard (wie Anm. 14), S. 277. 
protestantischen Fürsten blieb, zeigt sein Schreiben vom 19. Oktober 1592 an seinen Schwager Christian, Fürst von Anhalt, mit der Empfehlung für einen jungen Trompeter (Nr.15). Fürst Christian, der damals evangelische Einheiten im Krieg um das Straßburger Bistum führte, konnte diesen gut gebrauchen.

Ansonsten bieten die Schriftstücke aus St. Petersburg einen Einblick in den üblichen fürstlichen Alltag. Man bemerkt Mitleiden mit einem in Gefangenschaft geratenen Fürsten (Nr.12), erfährt in einem Kondolenzbrief vom Tod eines anderen Standesgenossen (Nr. 8). Ihren Platz finden aber vor allem Familienangelegenheiten: Die Verwandtschaft muss über die Verschiebung einer Hochzeit benachrichtet werden (Nr.5), Grüße und Glückwünsche werden geschickt (Nr.7). Auch die Jagd als fürstliches Plaisir fehlt nicht: Ein Leithund ist krank geworden, und die mit Ungeduld erwartete Hirschjagd ist in Gefahr. Jetzt soll ein befreundeter Fürst mit einem guten Leithund dienen (Nr.3). In einem anderen Schreiben wird für das übersandte Pferd gedankt (Nr.14) - ein zufällig überlieferter und zusammengewürfelter, aber durchaus charakteristischer Ausschnitt fürstlicher Lebenswelt im späten 16. Jahrhundert.

$$
\text { *** }
$$

\section{Editionen der Briefe}

Die nachfolgenden Transkriptionen der Briefe wurden buchstabengetreu entsprechend den Vorlagen erstellt. Die damals üblichen Interpunktionszeichen in Form von Schrägstrichen oder Punkten wurden übernommen, übliche Abkürzungen wurden stillschweigend aufgelöst, Ergänzungen erscheinen in eckigen Klammern.

1.

1552 Dezember 2, Pfullingen

Herzog Christoph von Württemberg an Graf Ludwig von Oettingen den Älteren: Der Graf von Oettingen soll ihn in etwa acht Tagen in Tübingen aufsuchen, vorher eine Auskunft über die Ausfertigung der Schriften an den Kaiser bei Dr. Caspar Volland und Dr. Nikolaus Varnbühler einholen. Das vom Grafen von Oettingen nachgefragte Datum im Vertrag zwischen dem Kaiser und dem Markgrafen Albrecht Alkibiades von Brandenburg-Kulmbach teilt Herzog Christoph mit (24. Oktober 1552).

WHA St. Pt. Institut f. Geschichte, 1/421.

Herkunft: erworben 1913 in Berlin beim Antiquariat Karl Ernst Henrici (Heinrici A XVIII, 17, 1913).

Eigenhändige Unterschrift des Herzogs. 1 Bogen 2', S.1-2 beschriftet. Briefverschluss durch unter quadratischer Papiertektur aufgedrücktes Sekretsiegel des Absenders. 
Außenadresse (Dem Wolgebornen vnnserm lieben Oheim vnnd besonndern Ludwigen dem Eltern / Grauen).

Vermerke: 1. Außenrubrum (M[ein] g[nediger] h[err] herzog Christoff v[on] Wurtemberg gibtt antwort [...] komen halb[en] Antritt den 4. Decembris etc 52). 2. Außenrubrum (Herzog Christoph zu Würtenberg An G[rafen] Ludwig[en] zu O[ettingen] etc den Eltern 1. nach ${ }^{16}$ Jnnerhalb 8. Tag[en] gehn Tübing[en] zu erscheinen. 2. bei D. Caspar Vollanden vnd D. Niclaus Varenbülern vmb Verferttigung der schrifft An die Röm: Kay: Mt: anzuhalten. 3. Bericht daß der Vertrag zwischen Kaÿ: Mt: vnd Marggraf Albr[echt] Den 24. ${ }^{\text {ten }}$ octob[ris] Anno 1552 zue seiner versöhnung / vfgericht. 2 Decemb[ris] Anno 1552).

Spätere Eintragungen mit Bleistift im linken Feld S. 1 (1008, vorhanden, 1552, Wirtemberg, Christophl, uno, 1552).

Vonn Gottes gnaden Christoff

Herzog zu Würtemberg etc

Vnnsern freuntlichen gruß zuuor / Wolgeborner lieber oheim vnnd besonder / Wir haben dein schreybenn deß Datum ist den letsten / ver / schines Monats Nouembris / empfangen / vnd seins Jnnhalltz verlesen Vnnd nach dem du Jnn einem eingelegten Zedell / anfangs melldest / das du dich / so es gerhaten sein wöllt / zu vnns verfüegen / vnnd Jnn diser sachen vnnsers Rhats pflegen wölltest etc Darauff füegen wir dir freundlicher maynung zuuernemen / Das wir jezmalls / vnnd noch der Zeyt vff dem waidwerck vmbziehenn / Aber vngeuarlich Jnnerhalb acht tagen ge / denncken wir (. durch Göttliche verlë̈hung .) widervmb ghen Tubingen zuuerruckenn / Alls dann magstu deiner gelegenhaÿt nach / dich zu vnns verfüegen / Wöllen wir dir vnnsers verstanndtz / allen freuntlichen vnnd getrewen Rhat mittaylen /

Es were auch gut / das du bey Doctor Caspar Vollanden / vnd Doctor Niclaus Varenbilern / vmb verfertigung der schrifften ann die Röm: Kay: Mt: etc vnnsern allergnedigsten herrn / anngehallten hettest / Dann vnns vonn Jnen noch der Zeÿt nichtz zukommen /

Nach dem du auch (. wie das Datum Jm Vertrag zwischen der Kay: Mt: vnd vnnserm freuntlich[en] lieben vettern Marggraf Albrechten zw Brandenburg etc stee .) begeren thust / haben wir denselbigen besichtigen lassen / Vnnd befindet sich daraus / das es der vier vnd zweinzigist tage deß Jungstverschinen Monats octobris ist / Welches alles wir dir vf gedacht dein schreyben freuntlicher maynung nit verhallten wöllen / Vnnd seind dir mit freuntlichem willen woll gewegenn / Datum pfulling[en] den 2. Decembris Anno etc .52.

Christoff Herzog zw Wirtemberg etc

16 Das Wort ist über der Zeile eingefügt. 
Herzog Christoph von Württemberg an Vogt, Bürgermeister und Gericht zu Bietigheim: Der Herzog verfügt die Erhebung der Kriegssteuer zugunsten der im Markgräflerkrieg geschädigten Mitglieder der Fränkischen Einigung.

WHA St. Pt. Institut f. Geschichte, 2/421.

Herkunft: erworben 1912 in Berlin beim Antiquariat Leo Liepmannssobn (Leo Liepmanssohn. Аукц. 40 Nering 9-11 дек 1912, N 357).

Eigenhändige Unterschrift des Herzogs. 1 Bogen $2^{\circ}$, S. $1-3$ beschriftet. Das unter quadratischer Papiertektur aufgedrückte Sekretsiegel des Absenders ist verloren, nur Reste des roten Wachses sind erhalten.

Außenadresse (Vnnsern vogt Burgenmaistern vnd Gericht zu Bietickhaim vnd lieben getrewen).

Vermerke: Außenrubrum (87 gulden v baz anlagg betreff[end] vff den 24. Septemb[ris] Anno Liiij).

Spätere Eintragungen mit dunkelbrauner Tinte im unteren Feld S. 1 (Herz. Christoph geb. d. 12 Mai 1515. Folgte seinem Vater, dem Herzog Ulrich 1550. Starb d. 28 Dez. 1568.) und hier auch das Datum mit brauner Tinte (1554).

Vonn Gotts gnaden Christoff

Herzog zu Wurtemperg etc

Vnnsern gruß zuuor Lieben getrewen. Wir geben Euch gnediger maÿnung zuerkhennen. Das Ro: Kaÿ: Mt: vnsers allergnedigsten herrs vnd dess hailigen Ro: Reichs Chamergericht zu Speÿr . marggraff Albrechtten zu Brandenburg etc. vor Jar vnd tagen Jn die ocht erkhendt . Auch Jr Kä̈: Mt: fur Jr Mt: selb . sollich ocht erclerung bestetigt. Vnd daruff den sechs Reichs Kraÿsen. Nemlich . den Vier Churfürsten am Rein . dem Reinischen . Paÿerischen . Schwäbischen . Frenckischen . vnd Obernsechssischen . die Execution . sollcher ochterclerung zum andern mal nachainandern . gar ernstlich gepotten . vnd darzu auch . zubezalung herzog hainrichs von Brounschweickh etc. Vnd boider Bischoff zu Bamberg vnd Wirzburg etc. Kriegsvalckhgelts . zum hechsten von nöten . So hat hechstgedachte Kä̈: Mt: an obgemelte. vnd sonst an all andre . dess Ro: Reichs kraißstend vnd also ouch an vns . aller gnadigst begert . den Frenckischen ainigungs verwanten stenden so Jn vergangnen kriegsemperungen grossen schaden erliten . Ain hilff mit gelt zuthun Doch mit der gnedigsten vertrostung . das Jr Mt: sollichs Jn kunfftigen gemainen Reichsanlagen . aller gnedigst . widerumb erstatten . oder ains ÿeden stands angepur. abziehen lassen wollenn Daruff die Churfursten vnd andre kraißstend . Sonderlich auch der Schwäbisch . ainhellig bewilligt . Das ain ÿeder Stand . ain ainfachen Rom Zug . vnd daran das halbthail . Jm ersten Monat . (.welcher sich vff den xxviij tag - yetzigs monats endet.) vnd den andern halbtheil . gleich Jm andern Monat hernach . erlegen soll. Dieweil Nun sollichs ain gemaine ordenliche Reichsanlag . dar Jnn gar Niemands verschont wurt. Vnd darzu allain ein sollich 
gelt ist. Welches Jn kunfftigen gemainen Reichs anlag widerumb soll erstattet oder abgezogen werden. Vnd herwiderumb wa das vff abbestimpte zwä̈ kurze zit nicht erlegt . Das wir. Auch vnsere prellaten deßgleichen getrewe Landschafft . vnd also auch Jr. gegen hechstgedachter Kaÿ: Mt: ainer grossen schweren Vngnad vnd achterclerung. Auch darnebenn ains gewaltigen vberfals vnd herzugs in vnser Furstentumb . Sonderlich aber . vonn Herzog Hainrichen von Bronschweickh . (.so dieser zeit mit aim Namhafften kriegsvolckh . zuRoß vnd fuß verfast ist . Jn bedenckhung . das die mandierten kraiß . die achterclerung . nit valnzogen Auch sonst allerhandt . hochbeschwerlicher weiterung · gewißlich gewertig . sein müssen. So haben wir vns hierJnnen . mit nichtenn . vnd khains wegs absundern . oder sollichs abschlahen khunden . Vnd gepurt also an sollichem ainfachen Römer Zug vnsern prellathen vnd gemainer Landschafft . Jn gemain . Zehen Thusent Neuen hundert Sechzig vnd Acht guldin Vnd Euch Jn Statt vnd Ampt . daran zuerlegen . wie Jr auß beiligendem zedel . vernemen werden. Vnd ist dem allem noch. Auch in betrachtung . abgehorter Eehafter tringender Vrsachen Vnser gnediger beuelch . Das Jr vnuerzug . gedacht Ewer angepur . Jn stat vnd Ampt / einpringen. Vnd vff die bestimpten zway Zit . allhieher . den verordneten schickhenn . damit sallich gelt . alßbald gen Speir erlegt . vnd also allerlaÿ gefar auch Nachtail verhiet werden mög. Verlassen wir vns gnediglich vnd ernstlich. Datum Stutgarten . den $x^{\text {ten }}$ Septembris Anno etc 54.

Cristoff herzog zw Wirtemberg etc

3.

1556 Mai 16, Blamont

Graf Georg von Württemberg und Mömpelgard an Graf Ludwig von Oettingen den Älteren: Graf Georg bittet um einen Leithund.

WHA St. Pt. Institut f. Geschichte, 5/421.

Herkunft: erworben 1911 in Leipzig beim Antiquariat List und Francke (L[ist] $\mathrm{u}$ [nd] Fr[ancke] Verst[eigert] 6.-8. November 1911, № 209). Stammt von der E[duard] F[ischer] v[on] R(öslerstamm) Autogr. Sammlung (Lit. A Nr. 988).

Eigenhändige Unterschrift und ein eigenhändiges Postscript des Grafen. 1 Bogen $2^{\circ}$, S. 1 beschriftet. Briefverschluss durch unter quadratischer Papiertektur aufgedrücktes Sekretsiegel des Absenders.

Außenadresse (Dem Wolgebornen vnnserm Lieben Oheim Ludwigen dem Eltern Grauen zu Ottingen).

Vermerke: 1. Außenrubrum (G[raue] Jorg v[on] Wurtemberg schreibt vmb ain laithund pre[s]entiert den 24 May gen Alerhaim $\mathrm{A}[\mathrm{nno}]$ [15]56). 2. Außenrubrum (Ain seer gnediger anmutiger brief).

Vnnser freundschafft zuuor wolgeborner lieber Oheim nachdem wir zu vorsteender Hirschfeiste mitt einem leithundt vbel versehen vnnd Jnn manngel steen, 
aber dannocht an solcher kurzweil nit gern verhinndert wirden wolten, So ist vnser ganz freundtlichs ansinnen vnd bitt an euch, Jr wolt wa muglich vnd Jr denselben nit hettet, aber bei euwern gutten herren vnnd freunden, bekommen mögt vns von altter kundschafft wegen ein gutten angeenden leithundt so von gutter art vnd zu gebrauchen sein möcht mittheilen, bei zeigern diß vnnsern derwegen zu geschickt Jagermeister zu kummen, vnd als einen altten son vatterliche treuw, erzeigen vnd daran nitt lassen / möchten seer wol leiden, wa die gelegenhait das geben möcht, Jr einmal bei vns kummen vnnd vnnser haushalttung auch besichtigen könnten Dann warum wir euch als vnnsern altten vatt[erlichen] freundtschafft vnd gutten willen zu erzeigen wüsten, sollt ir vns Jederzeit willig finnden Datum Blomont den xvj May A[nno] etc Lvj

Georg Graue zu Wurtemberg

Vnnd zu Mumppelgartt etc

G[eorg]. G[raue]. zu Wurttemberg etc

Lieber alte pater / so Es Euch gelucklich vnd mitt gutter gesuntheit wol zustundt hort Jch Götz Gern hab Lang Zeitt nicht von Euch vernomen / dz macht aber wan die heren wider Jn grossen Standt komen / So acht man der kleinen frundt vnd Sön nicht / nicht döster weniger so ich noch deinstlich könt sein zu ein Erlich[en] Kupler wie ich Ein mal mit Euch gerödt hab / So erbut mich diß willigs wer Grosse zeit daß jr Ein mal auch frümer wurd[en] den Grobe Rust schwab[en] vnd Getruwen Eidtgnoß / dem Lutz Euwerm Son minem Geuatter vn[d] geuaterin wolt vo[n] ${ }^{17}$ minett wäge vil guter zeit vn[d] alles gutz anzeig[en] /

Graf Georg von Württemberg-Mömpelgard an die hessischen Räte Friedrich von der Tann und Konrad Zollern von Speckwinckel: Graf Georg erinnert an den ausständigen Revers, die Herrschaft Reichenweier betreffend (Abb. 1).

WHA St. Pt. Institut f. Geschichte, 1/420.

Eigenhändige Unterschrift des Grafen. 1 Bogen 2, S. 1 beschriftet. Briefverschluss durch unter quadratischer Papiertektur aufgedrücktes Sekretsiegel des Absenders.

Außenadresse (Vnnsern lieben Besonndern Friderich von der Tann vnnd Conradt Zollern von Speckswinckel sampt vnnd sonders).

Georg Graue zu Würtemberg

Vnnd zu Mümppelgart etc

17 Die folgenden Worte sind im linken Feld geschrieben, da sonst der Platz für sie fehlte. 


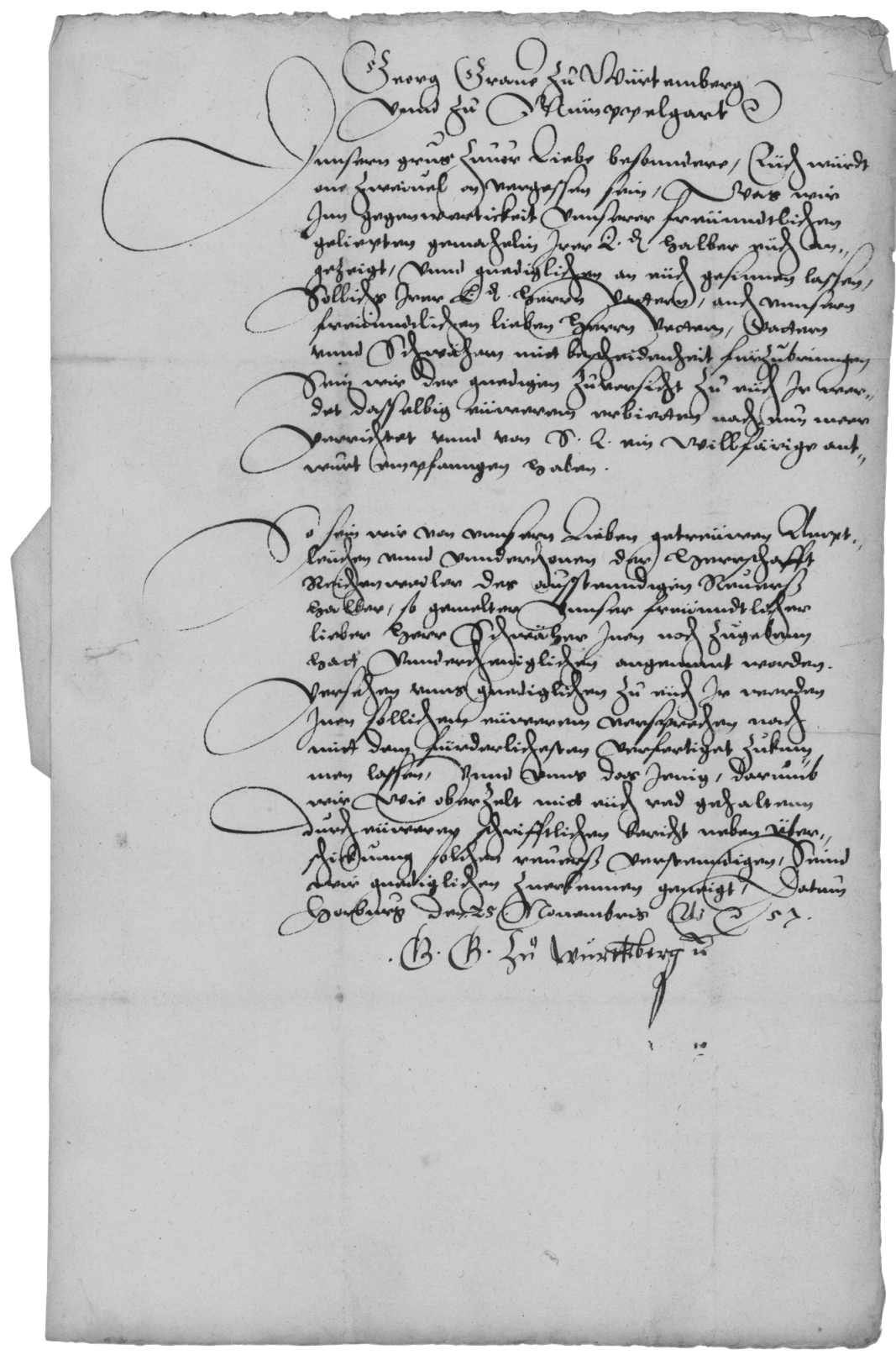

Abb.1: Brief Graf Georgs von Württemberg-Mömpelgard vom 25.11.1557 (Vorlage: WHA St. Petersburg 1/420). 
Vnnsern grus zuuor Liebe besonndere / Eüch würdt One Zweiuel on vergessen sein / Was wir Jnn gegenwertickeit vnnserer freünndtlichen geliepten gemahelin Jrer L[ieb] ${ }^{\text {den }}$ halber eüch angezeigt / vnnd gnediglichen an eüch gesinnen lassen / sollichs Jrer L[ieb] ${ }^{\text {den }}$ Herrn Vattern / auch vnnsern freunndtlichen lieben Herrn Vettern / vattern vnnd Schwähern mitt bescheidenheit fürzubrinngen Sein wir der gnedigen zu versicht zu eüch Jr werdet dasselbig eüwerem erbietten nach nun meer verrichtet vnnd von S[einer] L[iebden] ein willfärige antwurt empfanngen haben.

So sein wir von vnnsern Lieben getreüwen Amptleüthen vnnd vnnderthonen der Herrschafft Reichenweiler des ausstenndigen Reuerß halber / so gemelter vnnser freünndtlicher lieber Herr Schwäher Jnen noch zugebenn hatt vnndertheniglichen angemant worden. Versehen vnns gnediglichen zu eüch Jr werden Jnen sollichem eüwerem versprechen nach mitt dem fürderlichesten verfertiget zukummen lassen / vnnd vnns das Jenig / darumb wir wie oberzelt mitt eüch vnd gehaltenn Durch eüweren schrifftlichen bericht neben vberschickunng solchen reuerß verstenndigen / Seind wir gnediglichen zuerkennen geneigt / Datum Horburg den 25. Nouembris Anno etc 57.

G[eorg] G[raue] zu Württemberg etc

5. 1565 Dezember 24, Stuttgart

Herzog Christoph von Württemberg, an seinen Schwager Georg Friedrich Markgraf von Brandenburg-Ansbach: Christoph teilt die Verschiebung der Hochzeit seiner Tochter Sabine mit Landgrafen Wilhelm von Hessen mit (Abb. 2).

WHA St. Pt. Institut f. Geschichte, 6/421

Eigenhändige Unterschrift des Herzogs. 1 Bogen 2', S. 1 beschriftet. Briefverschluss durch unter quadratischer Papiertektur aufgedrücktes Sekretsiegel des Absenders. Eigenhändiges Postscript auf beigelegtem kleinen Blatt 21,9 $7,8 \mathrm{~cm}$.

Außenadresse (Dem hochgebornnen fursten Vnserm freundlichen lieben Oheimen Schwagern vnd geuattern Hern Georg Friderichen Marggrauen zu Brandenburg zu Stetin pomern der Cassuben vnnd wenden / auch in Shlesien zu Jagerndorff vnnd etc Herzogen Burggrauen zu Nurmberg vnnd Fursten zu Ruegen).

Vermerke: Präsentatum (praesentatum 6 Januarij 1566); Außenrubrum (Herzogk zw Wirtembergk zaigt ... ${ }^{18}$ seiner f[ürstlichen] g[naden] Tochter / frawlain Szabina Hochzait vff den xj. februarij erstregkt worden).

Spätere Eintragungen mit brauner Tinte im unteren Feld S. 4 (g 1515 reg: 1550 st. 1568).

Vnser freundlich dienst vnnd was wir liebs vnnd gutz vermögen / allezeit zuuor Hochgebornner furst / freundlicher lieber Oheim / Schwager / vnnd geuatter / Wir geben E[uer] L[iebden] freundlich zuerkhennen wiewol vnser Tochter hochzeit-

18 Ein Wort ist ausradiert. 


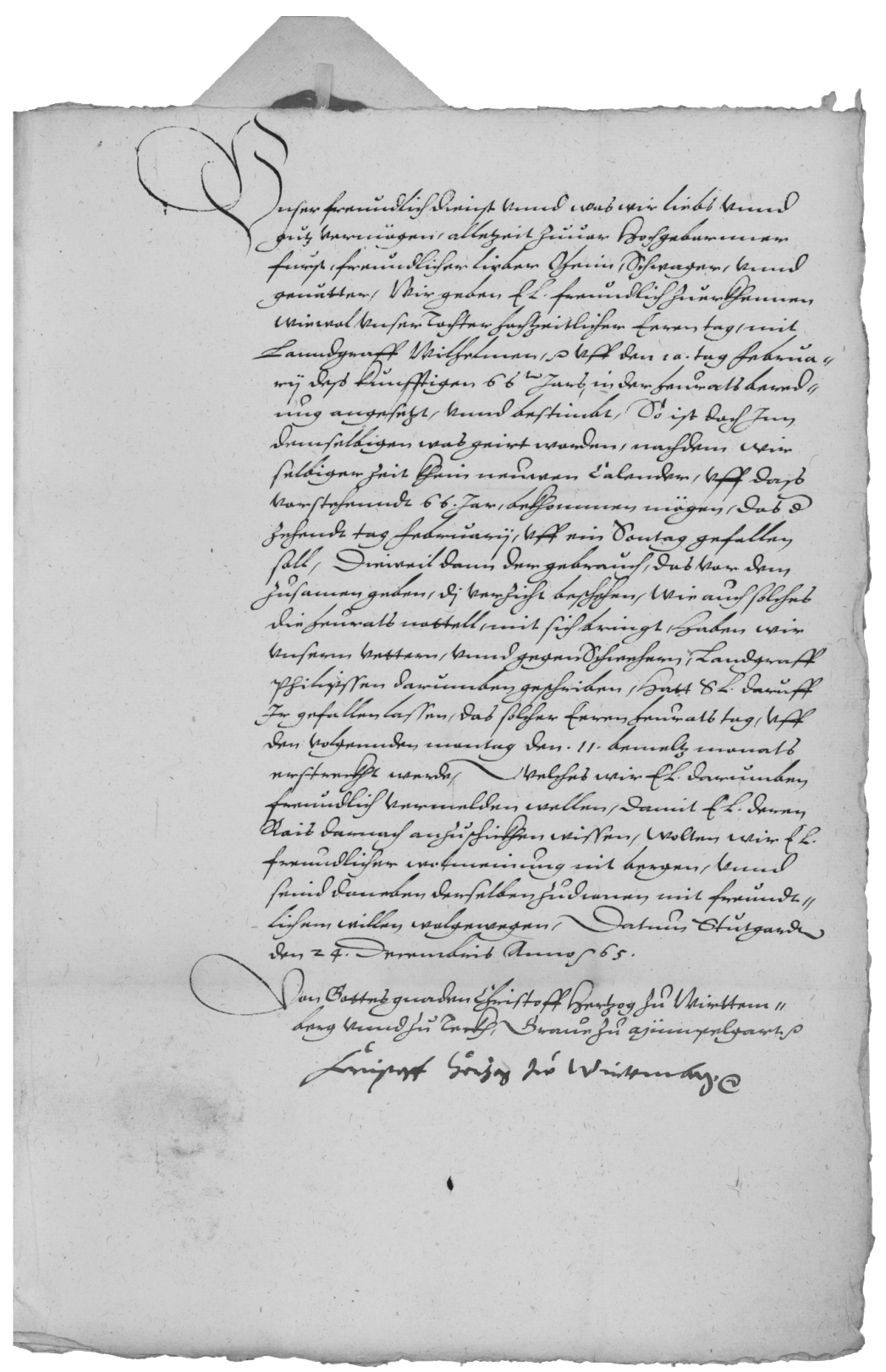

Abb.2: Brief Herzog Christophs von Württemberg vom 24.12.1565 (Vorlage: WHA St. Petersburg 6/421). 
licher Eeren tag / mit Lanndgraff Wilhelmen / etc vff den 10. tag Februarij des kunfftigen $66^{\text {ten }}$ Jars in der heurats beredung angesetzt / vnnd bestimbt / So ist doch Jnn demselbigen was geirt worden / nachdem wir selbiger zeit khein neuwen Calender / vff dass vorstehendt 66. Jar / bekhommen mögen / das d[er] zehendt tag Februarij / vff ein Sontag gefallen soll / Dieweil dann der gebrauch / das vor dem zusamen geben / dj verzicht beschehen / wie auch solches die heurats nottell / mit sich bringt / haben wir vnserm vettern / vnnd gegen Schwehern / Landgraff Philipssen darumben geschriben / Hatt S[eine] L[iebden] daruff Jr gefallen lassen / das solcher Eeren heurats tag / vff den volgennden montag den . 11. bemeltz monats erstreckht werde / Welches wir E[uer] L[iebden] darumben freundlich vermelden wellen / damit E[uer] L[iebden] deren Rais darnach anzuschickhen wissen / wolten wir E[uer] L[iebden] freundlicher wolmeinung nit bergen / vnnd seind daneben derselben zu dienen mit freundtlichem willen wolgewegen / Datum Stutgardten den 24. Decembris Anno etc 65.

Von Gottes gnaden Christoff Herzog zu Wirttem //

berg vnnd zu Teckh / Graue zu Mümpelgart etc

Cristoff herzog zw Wirtemberg etc

Post Scripta was vnns für Zeitung[en] auß Rom zukommen / Dauon thuen wir $\mathrm{E}$ [uer] L[iebden] hiemit Abschrifften freundtlich vnnd vertrewlich zu sennden etc Actum vt in Litteris.

6.

1567, Juli 21, Stuttgart

Herzog Christoph von Württemberg an Georg Friedrich Markgraf von Brandenburg-Ansbach, betreffend die Leuchtenbergischen Anforderungen an den Markgrafen von Brandenburg-Ansbach.

WHA St. Pt. Institut f. Geschichte, 7/421.

Herkunft: erworben 1899 in Leipzig beim Antiquariat List und Francke (List und Francke, cat. 302, N 1634.).

Eigenhändige Unterschrift des Herzogs. 1 Bogen 2', S. 1 beschriftet. Das für den Briefverschluss ursprünglich benutzte Sekretsiegel des Absenders unter quadratischer Papiertektur ist ausgeschnitten, Spuren des roten Wachses sind vorhanden.

Außenadresse (Dem Hochgebornen Fursten / vnserm freundtlichenn Lieben Oheim / Schwager vnd Geuattern / Herrn Georg Friedrich Marggrauen zu Brandenburg / zuo Stetin / Pommern / der Cassuben vnd Wenden / auch Jn Schlesien $\mathrm{Zu}$ Jägerndorff vnd etc Herzogen / BurgGrafen zu Nurmberg / vnd Fursten zu Rugen /).

Vermerke: Präsentatum (praesentatum den 23: Julij Anno etc 67:); 1. Außenrubrum (dess Herzogen Christoffen zu Wirttenbergs recepisse / sambt vberschickung etzlicher Newen Zeittung.). 2. Außenrubrum (Jtem bet[reffend] Vnterhandlung Jn des Landgrauen zu Leuchtenbergk schuldtsach). 
Spätere Eintragungen mit brauner Tinte im oberen Feld S. 1 (N 2, 286); mit Bleistift im unteren Feld S. 1 (Württemberg Christoph).

Vnnser freundtlich dienst / auch was wir liebs vnnd guts vermögen allzeit zuuor / Hochgebornner Furst freundtlicher lieber Oheim / Schwager vnd Geuatter / Wir haben E[uer] L[iebden] zwaj vnnderschiedliche schreiben / bede de datis den 15. diß noch werenden Monats / an gesstern zue vnsern hannden wol empfangen.

Souil nun die leuchtenbergisch anuorderung belangt / dieweil E[uer] L[iebden] vns nochmalen zu einem vnderhendler leiden mögen / So wöllen wir / so bald wir verstendigt vnnd bericht werden / das die Jung Leüchtenbergisch herrschaft veruormundet worden ist / begerter massen tag gehn Onolzpach furnemmen / vnd denselben bederseitz bej zeiten zuschreiben / die Jren zu solcher guetlichen vnderhandlung wissen zuuerordnen.

Am andern / So ist vnns deß bewüssten berichtz halber / den wir E[uer] L[iebden] vnderm dato den $4^{\text {ten }}$ huius Jn vertrawen zugesandt haben / vntz heer / weitter nichtz einkommen / was vnns aber derwegen weitter anlangen wurdet / das soll E[uer] L[iebden] vnser habenden Correspondenz nach / auch vnuerhalten bleiben.

Was vnns aber sonsten fur allerhandt zeittung angelangt hat / dauon lassen wir $\mathrm{E}$ [uer] L[iebden] zu continuierung angeregter Correspondenz / hieneben abschrifften freuntlich zukommen /

Vnd wollten solches E[uer] L[iebden] hinwider zu einem recepisse freuntlicher wolmeinung nit bergen / Vnnd derselben zudienen seien wir Jeder Zeit gutwillig / Datum Stutgarten den xxj ${ }^{\text {ten }}$ Julij Anno etc Lxvij .

Von Gottes gnaden Christoff herzog zu Würtem //

berg vnd zu Teckh / Graue zu Mumpelgart etc

Cristoff herzog zw Wirtemberg etc

7.

1568 Dezember 2, Stuttgart

Herzogin Anna Maria von Württemberg, Gemahlin von Herzog Christoph, an ihren Bruder Georg Friedrich, Markgraf von Brandenburg-Ansbach: Sie schickt Glückwünsche zum Neuen Jahr sowie eine Pelzdecke als Geschenk.

WHA St. Pt. Institut f. Geschichte, 3/421.

Herkunft: erworben 1912 in Berlin beim Antiquariat Leo Liepmannssobn (Leo Liepmanssohn. Аукц. 40 Nering 9-11 дек 1912, N 358).

Eigenbändiger Brief. 1 Bogen 2', die zweite Bogenbälfte feblt, für den Brief wurde nur ein Blatt benutzt. S.1-2 beschriftet. Das für den Briefverschluss ursprünglich benutzte Sekretsiegel der Absenderin ist nicht erbalten.

Die Außenadresse ist großenteils ausradiert ([...] hochgebornem [...] hern [...] zu seinen lieb handen).

Vermerke: Außenrubrum (die herzogin zu wurtenberg schickt Pelz deck zum neuen jahr). 
Hochgeborner furst freundlicher lieber vetter bruter vnd sunst e[uer] l[iebden] sein zuuor mein geburlich dinst vnd was ich in eren liebes vnd gutes vormach das sie e[uer] 1[iebden] von mir zuuor prad aus e[uer] 1[iebden] schreiben habe ich mit fraden vornumen das e[uer] 1[iebden] sampt e[uer] 1[iebden] gemachel meiner lieben dochter gesund sein got der almechtich wole e[uer] 1[iebden] peden noch lange zeit also erhalten vnd alles gutes jn sell vnd leieb vorleien meinen herz lieben heren vnd gemachel mich vnd vnsere kinder vnd vetteren solen e[uer] 1[iebden] so in zimlicher gesvnd heit wisen got der almechtich vorleie ferner sein gotliche genad nach seinem gotlichen wilen vnd vnser aler selen haeill vnd die weill ich in erfarung pin das e[uer] 1[iebden] ichstund auf dem schwein haz ist vnd ich meines pehaltes alhi von e[uer] 1[iebden] gehörd habe das e[uer] 1[iebden] kann dechped muth leiden so schicke ich e[uer] 1[iebden] himit zum ainem gluck selichen neuen iar an pelz decken ober e[uer] [ [iebden] die selbich von leich pas kund leiden als an deck ped vnd solle an got wel in ainer kurze an deck pedlein nacher kummen das ich hofe e[uer] 1[iebden] sole das selbich auch leiden kunnen mein herzen lieber her vnd gemachel vnd vnsere kinder lasen e[uer] 1[iebden] ale eren liebes vnd gutes sachen vnd so fere e[uer] 1[iebden] noch pein meinen geliebten bruter sein so pit ich e[uer] l[iebden] wolen mein fuos pot sein vnd seiner liebe mein schwesterliche liebe vnd drein ale eren liebes vnd gutes sachen damit sein sun e[uer] l[iebden] got dem almechtichen pefolen vnd ich pefilich mich e[uer] 1[iebden] in eren als meinen lieben vetteren pruder vnd sun datum stugarden den 2 dezember im 68 iar

ana maria herzogin

zu wirdenberg etc

8.

1573 April 6, Stuttgart

Herzog Ludwig von Württemberg, an Statthalter und Räte zu Weimar: Er kondoliert zum Tod Herzog Johann Wilhelms von Sachsen.

WHA St. Pt. Institut f. Geschichte, 7/420.

Herkunft: erworben 1899 in Leipzig beim Antiquariat List und Francke (List und Francke, cat. 302, N 1635.).

Eigenhändige Unterschrift des Herzogs. 1 Bogen 2, S.1-2 beschriftet. Das für den Briefverschluss ursprünglich benutzte Sekretsiegel des Absenders unter quadratischer Papiertektur ist nicht erhalten, Spuren des roten Wachses sind vorhanden.

Außenadresse (Den Edlen vnnd Hochgelerten / vnsern lieben Besonndern / N. Sächssischen verordneten Stathalter vnnd Räthen zue Weiwmar . / .).

Vermerke: Spätere Eintragungen mit Bleistift im unteren Feld S.1 (N 4122, Württemberg Ludwig III der Fromme, B.S.).

Von Gottes gnaden Ludwig

Herzog zue Württemberg etc 
Vnnsern günstigen grus zuuor / Edle / vnnd Hochgelerte / liebe Besonndere / Wir haben Ewer schreiben / de Dato / den 2 tag / des nechstuerganngen Monats Martij / an gestern / zue vnnsern hannden wol empfanhen / Vnnd daraus den tödtlichen abgang weilundt des Hochgebornnen fursten vnsers freundtlichen lieben Oheims vnnd schwagers / Herzog Hanns Wilhelmen zue Sachssen etc Gott seliger Milter gedechtnus / mitt betrübtem gemüet Aber herwider / sonnders gern vernommen / das S[einer] L[iebden] dermassen / nach langwiriger erdulter schwacheit / auß disem Jamerthal seliglich verstorben ist / Gott der herr / welle S[einer] L[iebden] an dem grossen tag / wie vnns dann nitt Zweifelt / Seittenmahl S[einer] L[iebden] Jn Zeit deren lebens / so Gott seliglich Regiert / vnnd gelebtt / auch Christenlich abgeschieden ist / mitt allen anndern außerwälten / ein frewdenreiche vrstenndt / gnediglich vnnd vätterlich mitthailen / Vnnd ab wol wir alle sterblich / So tragen wir dannacht mitt S[einer] L[iebden] hinderlassen gemählin etc auch Jungen herrschafft / vnnd der loblichen Landschafft / ein Christenlichs vnnd freundtlichs / auch gnedigs mittleiden / weil vnnd aber solches Gottes will / vnnd verordnung ist / So soll man auch dasselbig seiner Almacht beuelhen / vnnd haimbgeben / der welle auch zue dessen Glorj vnnd Eer / ermelte von Sachssen wittib / beneben der Jungen Herrschafft / bej langem leben / vnnd guttem fridlichen Regiment frissten vnnd erhalten / Wo auch wir Jren L[iebden] oder dero Lanndt vnnd leuthen / freundtlichen / gebuerlichen vnnd schwägerlichen dienst / auch euch günstigen vnnd gnedig[en] willen erzaigen vnnd beweisen könnden / wellen wir darzue freundtwillig / vnnd mitt gnaden genaigt erfunden werden / Wolten wir euch / hinwider zue günstiger / auch gnediger anntwort / nitt bergen / Datum Stuettgardten / den $6^{\text {ten }}$ Apprillis / Anno etc 73.

L[udwig]H[erzog]z[u]Württemberg etc.

$\mathrm{M}[\mathrm{anu}] \mathrm{p}$ [ro]pria s[ubscrip]s[i]t.

9.

1525 Februar 25, Stuttgart

Herzog Ludwig von Württemberg an einen Geistlichen fürstlichen Standes, betreffend die Renovation einer landesherrlichen Instruktion.

WHA St. Pt. Institut f. Geschichte, 8/421.

Eigenhändige Unterschrift des Herzogs. 1 Bogen $2^{\circ}$, die zweite Bogenhälfte mit der Adresse fehlt, für den Brief wurde nur ein Blatt benutzt. S.1-2 beschriftet. Briefverschluss durch unter quadratischer Papiertektur aufgedrücktes Sekretsiegel des Absenders.

Vermerke: Spätere Eintragungen mit schwarzer Tinte im unteren Feld S. 2 (Ludwig III. (od. V.) geb. d. 1. Januar 1554. Succed. 1571. + im Aug. 1593; S. Layritz, hist. geneal. Palmwald[er] etc.).

Vnnser frundtlich dienst / vnd was wir liebs vnnd guets vermögen Zuuor / Erwürdiger besonder lieber herr vnd frundt / E[uer] L[iebden] schreiben vmb 
mittaillung vnnser Newerungs Instruction / haben wir empfangen vnnd seins Innhallts vernommen / Vnnd ist gleichwol nit ohn / das vor Jarn der hochgeborn fürst herr Christoff herzog zu Würtemberg etc vnnser gnediger lieber herr vnd Vatter / millter vnd Christseliger gedechtnus / ein begriff einer instruction fassen vnnd Jn schrifften bringen lassen / Solche aber ist nach vnnsers Fürstennthumbs lannds art / hin vnd wider nach gelegennhait dessen zu mindern vnd zu mehrn gestellt / Auch allerlä̈ Eehafftin vnnd sonndere zugehördt gedachts Vnnsers Fürstenthumbs / die sich villeücht gegen anndern lanndsarten nit vergleichen . / .

Dieweil dann die Renouation so man zuthon begert / nach Jedes lanndsart reguliert werden soll / vnd dann vnnser Renouator / so E[uer] L[iebden] zu dero Renouation gebrauchen wellen / Allß der Jhenig so dannacht lanng darbey gewest / der sachen des Renouierens wol / vnnd Nun mehr einer bequemen Instruction / wie man Renouieren soll gnugsamb bericht / Sezen wir Jn keinen Zweifel er werde (. one vnnser Instruction .) an E[uer] L[iebden] vorhabenndt werckh des Ernewerns / nichs versomen / sonnder sich nach E[uer] L[iebden] lanndsart wol anzuschicken vnnd zu regulieren wissen /

Welches wir E[uer] L[iebden] vff deren schreiben / zu frundtlicher wideranntwurtung nit verhalten wellen / Seindt auch deren frundtliche dienst zuerzaigen sonders geneigt / Datum Stuttgardten den $25^{\text {ten }}$ Februarij Anno 1575

Von Gottes gnaden Ludwig herzog zu Würtem //

// berg vnnd zu Teckh / Graue zu Mümppelgardt etc

$\mathrm{L}[\mathrm{udwig}] \mathrm{H}[$ erzog]z[u]Württemberg etc

$\mathrm{M}[\mathrm{anu}] \mathrm{p}[$ ro]pria $\mathrm{s}[\mathrm{ubscrip}] \mathrm{s}[\mathrm{i}] \mathrm{t}$

10. 1581 November 6, Böblingen

Herzog Ludwig von Württemberg an die Kirchenräte zu Stuttgart: Diese sollen die beigefügten neuen Kirchenlieder prüfen und deren Autor dementsprechend bezahlen lassen (Abb. 3).

WHA St. Pt. Institut f. Geschichte, 10/421.

Eigenhändige Unterschrift des Herzogs. 1 Bogen $2^{\circ}$, die zweite Bogenhälfte fehlt, für den Brief wurde nur ein Blatt benutzt. S. 1 beschriftet. Das für den Briefverschluss ursprünglich benutzte Sekretsiegel des Absenders unter quadratischer Papiertektur ist nicht erhalten, Spuren des roten Wachses sind vorbanden.

Außenadresse (Vnnsern Kürchenräthen zue Stutgardten vnnd lieben getrewen).

Vermerke: Spätere Eintragungen mit Bleistift im unteren Feld S. 1 (Württemberg Ludwig der Fromme, Sohn Chr. +1593 ), mit brauner Tinte im oberen Feld S. 2 (f. 25.).

Von Gottes gnaden Ludwig

Herzog zue Württemberg etc 


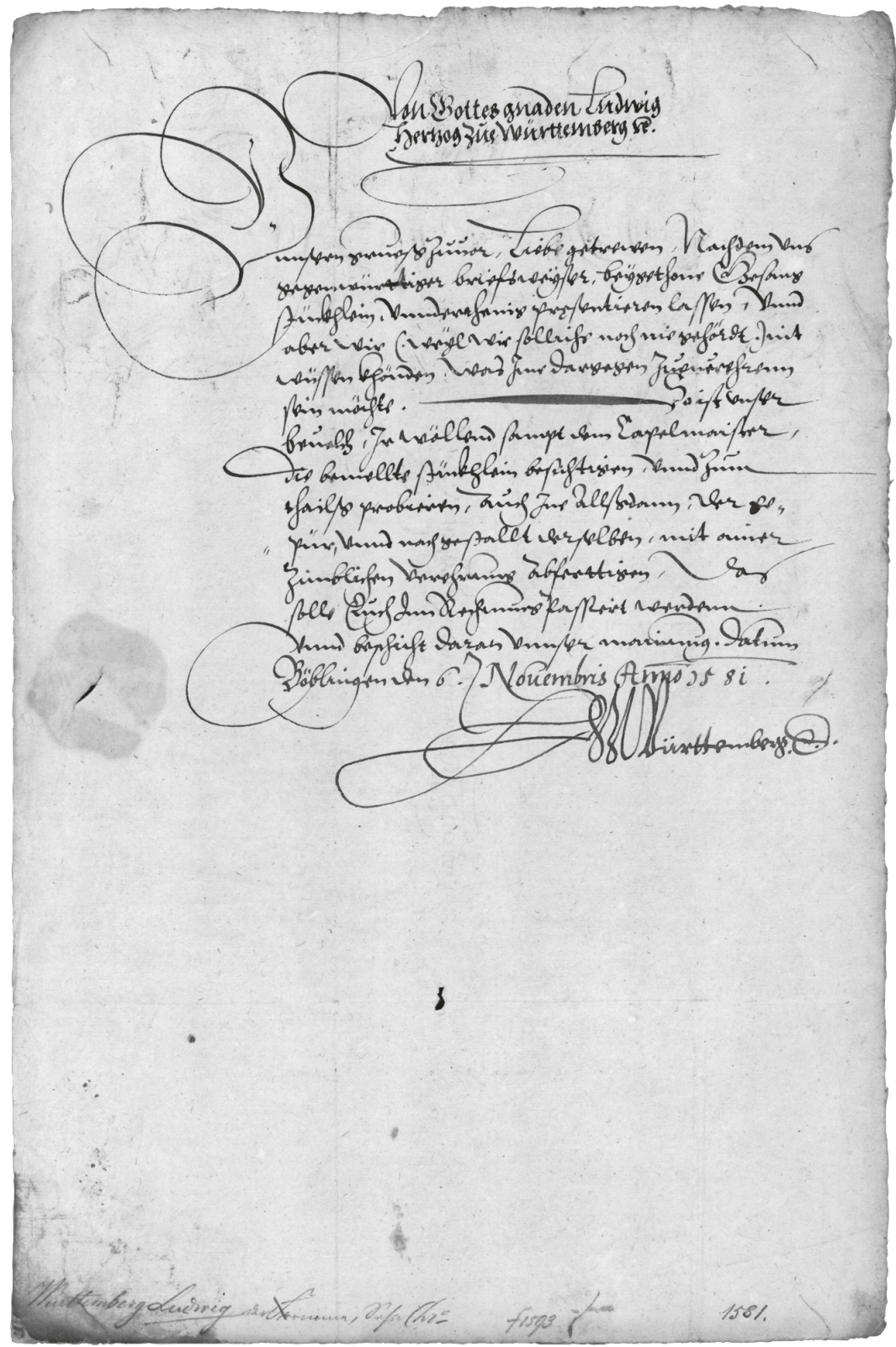

Abb. 3: Brief Herzog Ludwigs von Württemberg vom 6.11.1581 (Vorlage: WHA St. Petersburg 10/421). 
Vnnsen gruess zuvor / Liebe getrewen / Nachdem vns gegenwürttiger briefsweÿser / beÿgethone Gesang stückhlein / vnnderthenig presentieren lassen / Vnnd aber wir (. weÿl wir solliche noch nie gehördt .) nit wüssen khönden / was Jme dargegen zueuerehrenn sein möchte. So ist vnser beuelch / Jr wöllend sampt dem Capelmaister / die bemellte stückhlein besichtigen / vnnd zum thailss probieren / Auch Jne allssdann der gepür / vnnd nach gestallt derselben mit ainer zimblichen verehrung abferttigen / Das solle Euch Jnn Rechnung passiert werdenn vnnd beschicht daran vnnser mainung. Datum Böblingen den 6. Nouembris Anno 1581

$\mathrm{L}[\mathrm{udwig}] \mathrm{H}[\mathrm{erzog}] \mathrm{z}[\mathrm{u}] \mathrm{Württemberg}$ etc

11.

1585 April 28, Mömpelgard

Graf Friedrich von Württemberg-Mömpelgard an Schultheiß, Bürgermeister und Rat von Bern, betreffend die Wiedereinlösung der Grafschaft Valangin.

WHA St. Pt. Institut f. Geschichte, 12/421.

Herkunft: erworben 1911 in Leipzig beim Antiquariat List und Francke (L[ist] $\mathrm{u}$ [nd] Fr[ancke] Verst[eigert] 6.-8. Nov[ember] 1911, No 210). Stammt von der E[duard] F[ischer] v[on] R[öslerstamm] Autogr. Sammlung (Lit. A Nr. 986).

Eigenhändige Unterschrift des Grafen. 1 Bogen 2, S. 1-3 beschriftet. Das für den Briefverschluss ursprünglich benutzte Sekretsiegel des Absenders unter quadratischer Papiertektur ist nicht erhalten, Spuren des roten Wachses sind vorbanden.

Außenadresse (Den Fursichtigen Ersamen vnnd Weisen Vnsern Besondern lieben Nachbaurn vnd guetten Freunden N. N. Schuldtheiss Burgermeister vnd Rath der Statt Bern : / :).

Vermerke: Außenrubrum (Mümpelgart $\mathrm{p}[\mathrm{ro}]$ mothoriales zu gunst der gräfin vonn Challant). Spätere Eintragungen: mit Bleistift im oberen Feld S. 1 (Neuenburg, VI. I., 1585 April 28.); mit Bleistift im unteren Feld S. 1 (1585, Würtenberg, Friedr.); mit brauner Tinte im oberen Feld S. 4 (1585. 28. April.).

Von Gottes Gnaden Friderich Graue

zu Württemberg vnd Mümppelgartt etc

Vnnsern gunstigen grues zuuor / Fürsichtige / Ersame / vnnd Weise / besonndere liebe Nachbaurn / vnnd guette freundt / Nachdem wir / zugleich vnnsern Hochlöblichen Altfordern / des Hauß Württembergs etc. bej Euch ohne Vnnderlaß alle guette Nachbaurschafft / vnnd vertreuwliche Correspondenz bißher gespürt / vnnd derenthalben / vnns noch Jnn Künfftig einnichen Zweiuel nit machen / sie werden Jnn solchen guetten vertrauwen / weniger nit / dan wir / Zugleich Zu Continuieren geneigt sein / So khönnen wir Aus solchem gemeinen guetten Verstandt / Euch diser Zeitt Nachbeurlichen nicht verhaltten / Nämlich das wir / ohnlänngest / durch die wolgebornne Fraiw / Ysabella Greuin Zu Schallant etc. vff das aller flehenlichst ersucht / vnnd freundtlich gebetten / Jro / 
Vnd deren geliebten Kindern / Zum besten / Jnsonnderheit aber widerlösung der Grauenschafft Valangin / mit vnnserm beistannd / vnnd hilff / Auch vffbringung der somma geltt darumb Weilundt Jhr Herr / Vnnd Vatter / Rheinhardt Graue von Schalant sehliger / sie bej Euch versezt / verholffen zu sein / Wan Vnns nun / vß Allerhanndt vffgelegter briefflicher gewarsam Jn originalj / vnnsers behalz für genugsam erschienen / das sie billich wider zu dem Jhren gelassen werden soll / Auch Jnn ansehung / die Von Valangin / bej vnnserm Altt Fürst vnnd löblichem Hauß Mümppelgartt / Je vnnd Allwegen Jnn bestem angesehen / Vnnd sonnsten wolluerdient gewesen / zudem vnns auch noch / was Anforderung selbiger Herrschafft Valangin halben beuorsteth Jnmassen von Vnnsert wegen / durch Vnnsere Lannduogt / Canzler vnnd Räth / Anno etc 77 bej Euch / Vnnd einer Statt Solothurn anregung beschehen / Wir auch Euwere beantwurttung noch beihannden haben / Dannenhero wir wenniger nit / dan bej allen Fürstlichen / vnnd andern dergleichen gebornen Persohnen / Rhum vnnd Löblich (einannder Jnn obligenden ehafften sachen / vnnd nottfählen / mit hilff zuzuspringen) Jro auch auß freundtlicher Zuneigung vnnd Vermöglichait vnnsern beistanndt nit versagen wellen / So sein wir auch der guetten Vnnd freunndtlichen Zuuersicht / Ihr werden Euch / Alß denen Jr fueg am basten bekhanndt / vnnd die für sich selbß der billichait / woll gewagen sein / ein solches nicht frembdt sein / Wie wir dan nitgern Vnnsers wüssens / ettwas / so vnnser Vralt guet Vertrauwen / Vnnd Corresponndenz / freundt vnnd Nachbaurschafft / vmb das geringste endern möchte / fürnehmmen woltten / Sonder sie die Greuin / wie billich widerumb Vermittelst erstattung / der darumb vßgelegten Summa geltts / vnnd sonnsten was Recht vnnd billich / Vermög Euwerer selbß Verschreibung / vnnd deren Von Longewille gethonner Vbergab / $\mathrm{Zu}$ dem Jhren / khommen lassen / Auch Euch also erzeigen / wie wir zu Euch / vnnser ohnzweiuenliches freundtlich vnnd Nachbeurliches Vertrauwen tragen / Damit sie diser vnnserer erwissenen Freunndtschafft / vnnd fürbitt / bej Euch / genossen haben / Jm werckh spüren möge / Welches neben dem es billich vnnd der gerechtigkheit gemeß / Steth vnns vmb Euch / Jnn gleichem vnnd mehrerm / vff zutragenden fall / zubeschulden / Dan Euch allen freundt / vnnd Nachbeurlichen guetten willen / zuerzeigen / Habt Jhr vnnd All Zeit woll gewog[en] Dat[um] Jnn vnnserm Schloß Mümppelgartt den $28^{\text {ten }}$ Aprilis Anno etc 85 . Stilo Veteri .

Friderich Graue zu Wurtte[m]berg

Vnd Mümpellgardt etc

[Manu] propria s[ub]s[cripsi]t 
Herzog Ludwig von Württemberg an Wilhelm, Herzog von Bayern: Ludwig zeigt sein Mitleid mit Erzherzog Maximilian wegen der Niederlage im Kampf um den polnischen Thron (Abb. 4).

WHA St. Pt. Institut f. Geschichte, 9/421.

Eigenhändige Unterschrift des Herzogs. 1 Bogen 2, S. 1 beschriftet. Briefverschluss durch unter quadratischer Papiertektur aufgedrücktes Sekretsiegel des Absenders.

Außenadresse (Dem hochgebornnen Furssten Vnnserm freundtlichen lieben Vettern vnnd Bruedern / Hern Wilhelmen Pfalzgrauen bej Rhein / Herzogen Jnn Obern vnnd Nidern Bayern).

Vermerke: Präsentatum (Prä[sentatum] den $19^{\text {ten }}$ Februarij Anno etc 88); Außenrubrum (wirttemberg schikht zeitung vom Erzherzog Maximilian. N 278).

Spätere Eintragungen: 1. mit Bleistift im linken Feld oben S.4 (Würtemberg Herzog Ludwig übersendet Kriegs-Neuigkeiten. 1588.). 2. mit brauner Tinte im unteren Feld S. 4 (Ludwig der Fromme. g. 1554. succed: $1568+1593$ sine prole).

Vnnser freunndtlich diennst / Auch was wir liebs vnnd guetts vermögen Zuuor / Hochgebornner Fürsst / Freundtlicher lieber Vetter vnnd Brueder / . Was vnns heuttges Tags für Pollnische Zeyttungen einkommen / Thüeen wir E[uer] L[iebden] hergebrachter Vertrewlicher Correspondenz nach freundtlich Communiciern / . Da nun die Sachen mit vnnsers auch freunndtlichen lieben herrn Vetters Erzherzog Maximilianj etc L[au]t sollicher gestalldt beschaffen weren / Triegen wir desshalben ein Trewlich Vetterlich Mitleiden / Vnnd were Allßdann wol vonnötten / Das ettliche hohe Häupter dieser sachen sich güettlich vnnderfiengen / Damit beederseiz verrer Bluett vergiessen verhüettet wurde etc Wolten wir E[uer] L[iebden] zue vortpflannzung dess guetten vertrauwens / wie es An vnns gelanngt / freundtlicher Mainung nicht pergen / . Vnnd seindt dero angneme freundliche diennst zuerzeigen genaigt / . Datum Stuettgardt denn . 29 ${ }^{\text {ten }}$ Januarij Anno. 1588./.

Von gottes gnaden Ludwig herzog zue Württem //

// berg vnnd zue Teckh / Graue zue Mümppelgart etc .

$\mathrm{L}[\mathrm{udwig}] \mathrm{H}[\mathrm{erzog}] \mathrm{z}[\mathrm{u}]$ Württemberg etc

$\mathrm{M}[\mathrm{anu}]$ propria $s[\mathrm{ub}] \mathrm{s}[\mathrm{cripsi}] \mathrm{t}$ 


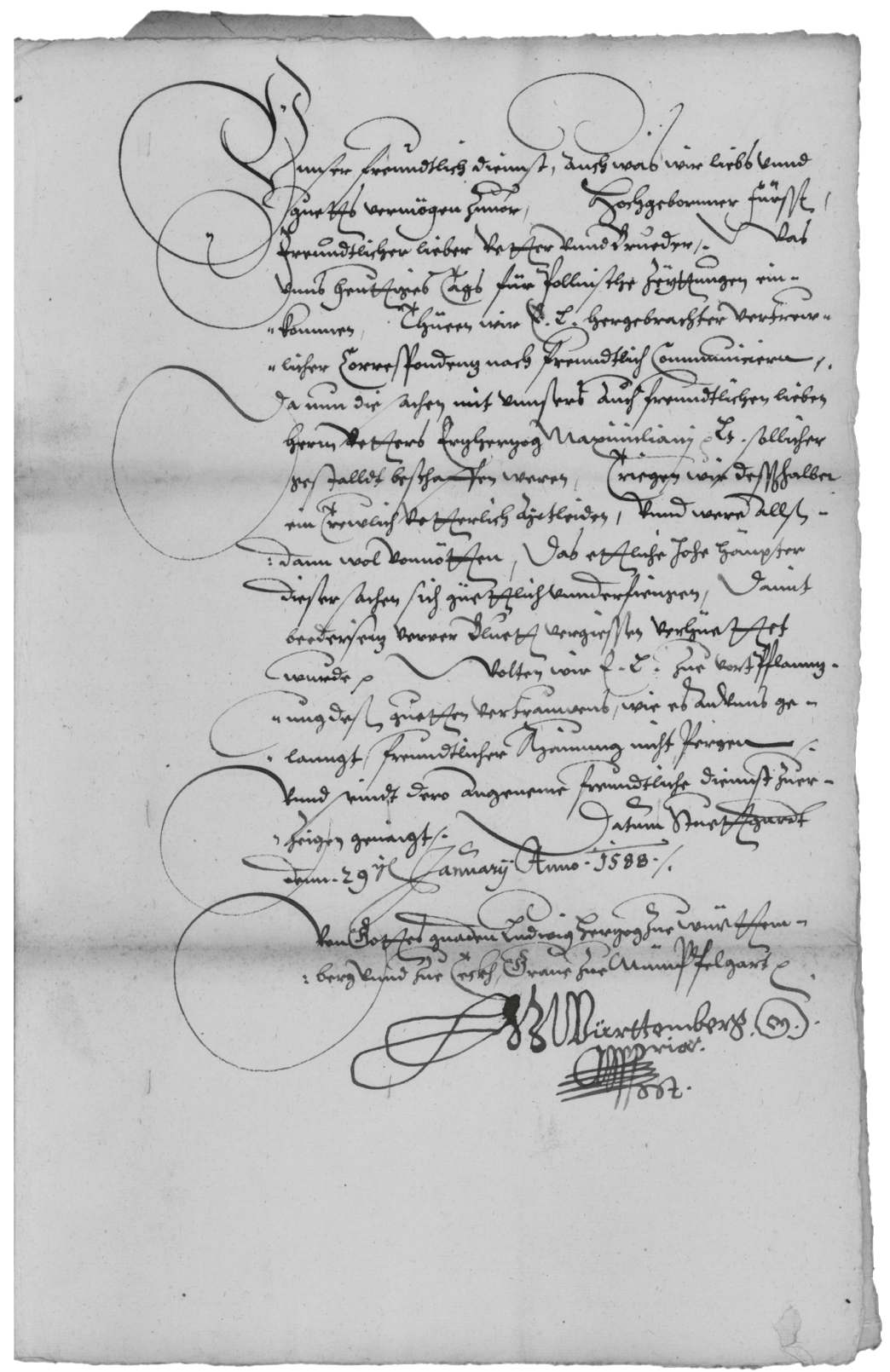

Abb. 4: Brief Herzog Ludwigs von Württemberg vom 29.1.1588 (Vorlage: WHA St. Petersburg 9/421). 
13.

1588 September 1, Blamont

Graf Friedrich von Württemberg-Mömpelgard an eine Person fürstlichen Standes: Er gibt einen Bericht über einen Überfall der Lothringer auf Mömpelgard von 1440 wieder (Abb. 5).

WHA St. Pt. Institut f. Geschichte, 15/421.

Eigenhändige Unterschrift des Grafen. Beschädigtes Fragment, die obere Hälfte des ersten Blattes $27 \times 17 \mathrm{~cm}$ von ursprünglich 1 Bogen $2^{\circ}$ ist erhalten. Das Fragment ist auf beiden Seiten beschriftet. Siegel und Adresse feblen.

Vermerke: Ziffer im linken Feld S. 2 (131 60; 13565).

Spätere Eintragungen: 1. Mit brauner Tinte im oberen Feld S. 1 (172.), im linken Feld S. 1 (1. Sept 1588). 2. Mit Bleistift S. 1 (Württemberg, Friedrich Graf. 12136).

Vnnser Freundtlich dienst / vnnd was wir liebs vnnd guetz vermögen AllZeit zuuor. Hochgebornner Fürst / freundtlicher lieber Herr Vetter vnnd Geuatter .

Dieser tagen haben wir neben annderm / Jnn vnnserer Registratur souil berichtz gefunden / wie das Anno 1440 durch den Lottringer fast ein gleichmessiger feindtlichen einfall / mit Rauben / Verhergen vnnd Annderm Jnn vnnsere Grauschafft Mumppelgartt beschehen / Alß E[uer] L[iebden] vß beigelegter Altten Missiuen zuuernehmmen / Welches wir E[uer] L[iebden] allein der Meynung verstendigen wellen / Damit sie wüssens haben möge / Das diß nicht das erste mahl / das Lottringen sich dergestalt feindtlich erzeigt / vnnd man genugsame . vnnd billiche vrsachen habe . die sach nicht ersitzen zulaßen / Sonder dermaln eins . sich wider zurachen / Sonsten dörfften sie einen bösen / vnnd PraeJudicirlichen habitum darauß machen

Beneben sendt wir Auch gleub [...] Vnnd entdtlichen beri [...] worden / Vie [...] vorhabens seindt $[\ldots]^{19}$

Vnnd wir bleiben E[uer] L[iebden] wie Allwegen zu Angenehmmen beheglichen diensten / willig vnnd wollgeneigt Dat[um] Inn vnnser Vestung Blaumont den Ersten Septembris Anno etc 88

E[uer] L[iebden] getrewer Vetter alzeitt

Friderich Graue zu Württemberg

vnd Mum[m]ppelgardt . etc

[Manu] propria s[ub]s[cripsi]t

19 Dieser Teil des Textes ist durch Abriss nur fragmentarisch erhalten. 

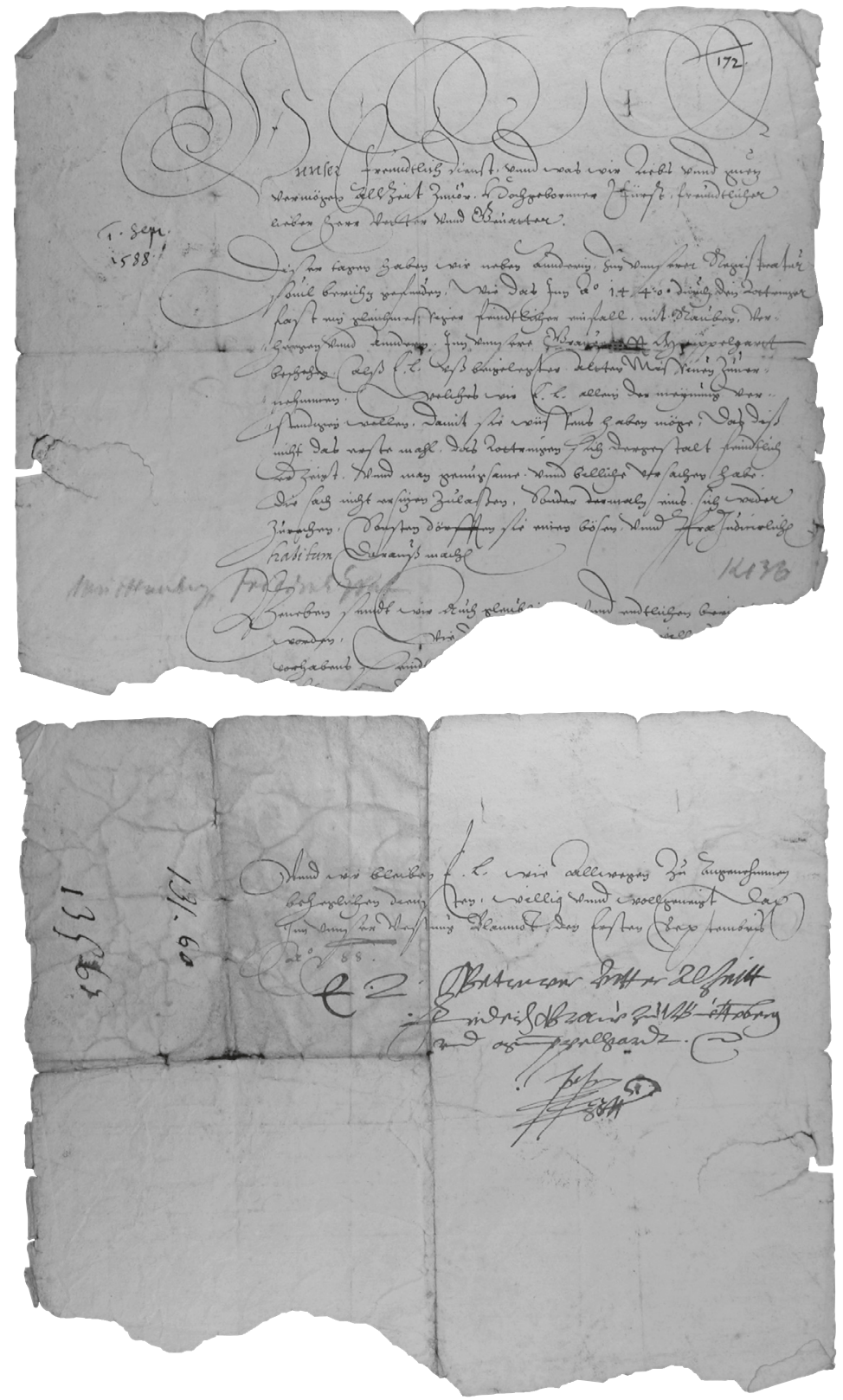

Abb. 5: Brief Graf Friedrichs von Württtemberg-Mömpelgard vom 1.9.1588

(Fragment, Vorder- und Rückseite; Vorlage: WHA St. Petersburg 15/421). 
14.

1589 Oktober 4, Stuttgart

Herzog Ludwig von Württemberg, an Grafen Gottfried von Oettingen: Der Herzog bedankt sich für ein ungarisches Pferd (Abb. 6).

WHA St. Pt. Institut f. Geschichte, 11/421.

Eigenhändige Unterschrift des Herzogs. 1 Bogen 2;, S. 1 beschriftet. Das für den Briefverschluss ursprünglich benutzte Sekretsiegel des Absenders unter quadratischer Papiertektur ist nicht erhalten, Spuren des roten Wachses sind vorhanden.

Außenadresse (Dem Wolgebornen vnnserm Oheim vnnd lieben besonndern / Gottfriden / Grafen zue Ottingenn . /.). Außendatum (4 Octobris 1589).

Vermerke: Spätere Eintragungen mit Bleistift im unteren Feld S. 1 (Württemberg Ludwig III Herzog genannt d. Fromme 1589).

Von Gottes gnaden / Ludwig Herzog / zue Württem //

// berg vnd Teckh / Graue zue Mümpelgartt. etc.

Vnnsern freundlichen grues zuuor / Wolgeborner / lieber Oheim vnnd Besonnnder . Wir haben das grawe Vngarische Pferdt / so du Vnnß bë̈ vnnserm abgeferttigten diener zuegeschickht hasst / Wol empfangen . Dasselbige ist vns sehr angenemb / vnd geraicht vnns von dir zue sonnderm gnedigem gefallen . Jnmaßen wir auch genaigt seÿen solches gegen dir in annderwege mit freundtlichem vnnd gnedigem willen zuerkhennen. Wollten wir dir (. Dem wir one das ganz wol gewogen /) zur danckhsagung freundtlich vnnd gnedig nit pergen. Datum Stuetgardten / den 4. Octobris. Anno. 1589.

L[udwig]H[erzog]z[u]Württemberg etc

15. 1592 Oktober 29, Granges

Graf Friedrich von Württemberg-Mömpelgard, an Christian, Fürst von Anhalt: Der Graf empfiehlt einen jungen Trompeter (Abb. 7).

WHA St. Pt. Institut f. Geschichte, 14/421.

Eigenhändige Unterschrift des Grafen. 1 Bogen 2, S. 1 beschriftet. Briefverschluss durch unter quadratischer Papiertektur aufgedrücktes Sekretsiegel des Absenders.

Außenadresse (Dem Hochgebornnen Fürsten / vnserm Frundtlichen lieben oheim vnndt Schwagern Herren Christian Fürsten zu Anhaltt / Grauen Zu Ascanien / Herren zu Zerbst Vnnd Bernburg etc).

Vermerke: Spätere Eintragung mit brauner Tinte im oberen Feld S. 1 (3).

Vnnser Freunndtlich dienst / vnnd was wir Liebs vnndt guetz vermögen Allzeit zuuor Hochgebornner Fürst freundtlicher lieber Oheim vnnd Schwager / Vnns hatt gegenwürttiger Zeiger Junger Angehennder Trommetter / Vnnder thennig Zuerkhennen geben / Wie das er bej Jtzigen guetten gelegenheitten / ettwas ferners Zuuersuechen / Zuerfahren / Vnnd sonnderlich E[uer] L[iebden] zu diennen 




Abb. 6: Brief Herzog Ludwigs von Württemberg vom 4.10.1588

(Vorlage: WHA St. Petersburg 11/421). 


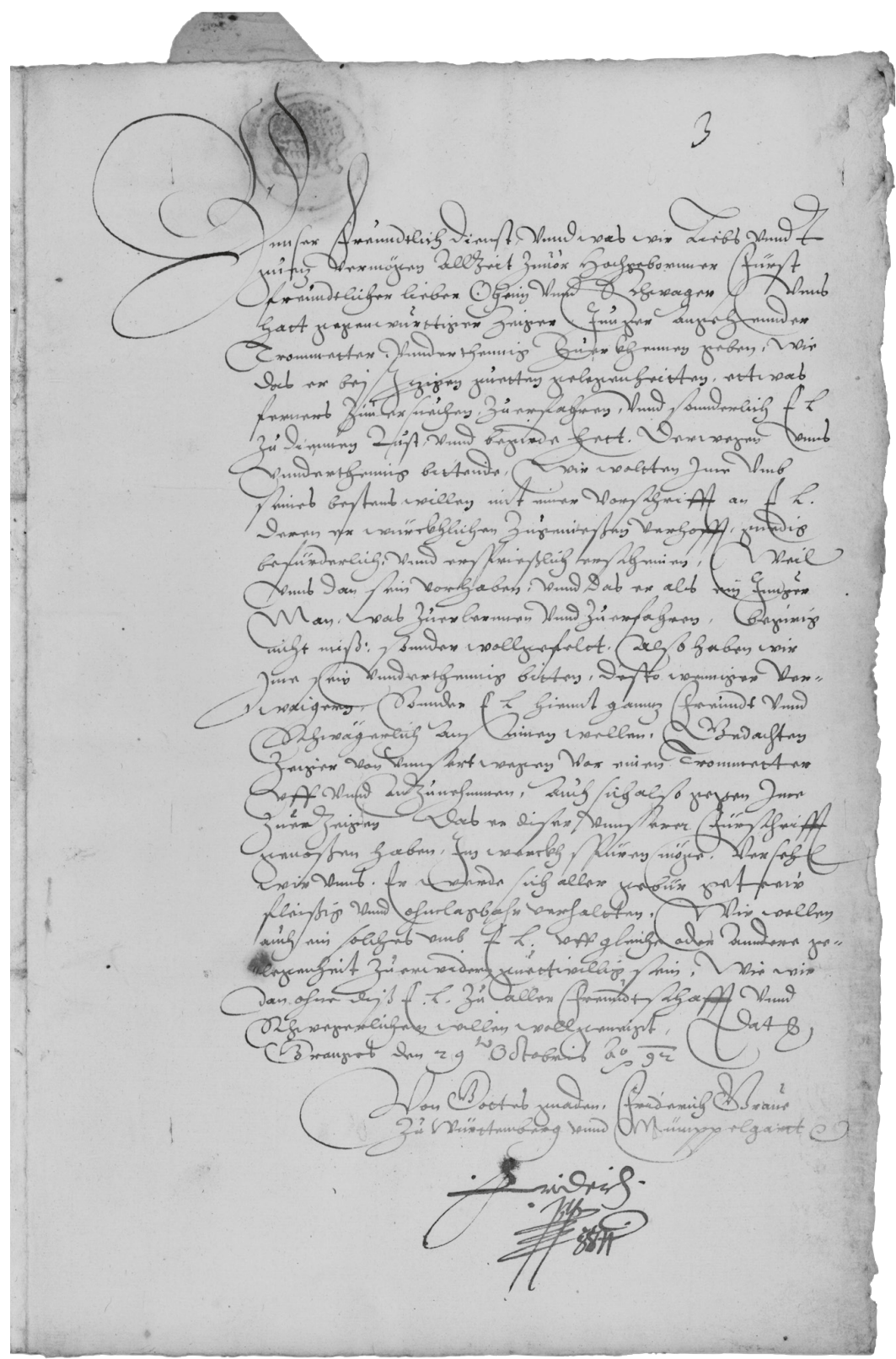

Abb. 7: Brief Graf Friedrichs von Württemberg-Mömpelgard vom 29.10.1592 (Vorlage: WHA St. Petersburg 14/421). 
Lust / vnnd begirde hett. Derwegen vnns vnnderthennig bittende / wir woltten Jme Vmb seines bestens willen mit einer Vorschrifft an E[uer] L[iebden] deren er würcklichen Zugenießen verhofft / gnedig befürderlich / vnnd ersprießlich erscheinen / Weil vnns dan sein vorhaben / vnnd das er als ein Junger Man / was zuerlernnen Vnnd Zu erfahren / begirig nicht miß / sonnder wollgefeltt / Also haben wir Jme sein Vnnderthennig bitten / desto wenniger Verwaigern / Sonnder E[uer] L[iebden] hiemit ganz Freundt vnnd Schwägerlich Ansinnen wellen / Gedachten Zeiger von vnnsert wegen vor einen Trommetter vff vnnd Anzunehmmen / Auch sich also gegen Jme Zuerzeigen Das er dieser vnnserer Fürschrifft genoßen haben / Jm werckh spüren möge / Vesehen wir vnns / Er werde sich aller gebür getrew fleißig vnnd ohnclagbahr verhaltten / Wir wollen auch ein solches vmb E[uer] L[iebden] vff gleiche oder Anndere gelegenheit zu erwidern guettwillig sein / Wie wir dan ohne diß E[uer] L[iebden] $\mathrm{Zu}$ allen Freundtschafft vnnd Schwegerlichem willen wollgeneigt / Dat[um] Granges den $29^{\text {ten }}$ Octobris Anno etc 92

Von Gottes gnaden / Friderich Graue

$\mathrm{Zu}$ Württemberg vnnd Mümppelgartt etc

Friderich [Manu] propria s[ub]s[cripsi]t 